\title{
Development of a short questionnaire to assess the dietary intake of heterocyclic aromatic amines
}

\author{
Sabine Rohrmann ${ }^{1,2, *}$ and Nikolaus Becker ${ }^{1}$ \\ 'Deutsches Krebsforschungszentrum, Division of Clinical Epidemiology, Im Neuenheimer Feld 280, D-69120 \\ Heidelberg, Germany: ${ }^{2}$ Present address: John Hopkins Bloomberg School of Public Health, Department of \\ Epidemiology, 615 North Wolfe Street, Room E6133, Baltimore, MD 21205, USA
}

Submitted 13 August 2001: Accepted 22 January 2002

\begin{abstract}
Objective: Development and validation of a short instrument to assess the dietary intake of heterocyclic aromatic amines (HCA).

Design: At first, a longer instrument asking for the consumption of 11 meat and fish items and different preparation methods was developed. The degree of browning of these foods was assessed by means of photos. This questionnaire was sent to 500 participants of the European Prospective Investigation into Cancer and Nutrition (EPIC) in Heidelberg, Germany, in June 1999. Using 385 completed questionnaires, a short questionnaire was developed covering just seven food items, which was sent to the participants again. Of these, 344 were returned within four months. Total dietary intake of HCA as well as the intake of different HCA were calculated and compared between both versions.

Results: Median dietary intake of total HCA was $103 \mathrm{ng}$ day $^{-1}$ as assessed with the short version; the intakes of 2-amino-1-methyl-6-phenylimidazo[4,5- $b$ ]pyridine (PhIP), 2-amino-3,8-dimethylimidazo[4,5- $f$ ] quinoxaline (MeIQx) and 2-amino-3,4,8trimethylimidazo[4,5- $f$ ]quinoxaline (DiMeIQx) were 63, 34 and $2 \mathrm{ng}$ day $^{-1}$, respectively. These results did not differ significantly from those obtained with the longer version. Spearman rank correlation coefficients between the long and the short version ranged from 0.46 to 0.6 . In quartile cross-classification, $70-78 \%$ of the participants were assigned into the same or an adjacent quartile while categorisation into opposite quartiles was $\leq 3.5 \%$.

Conclusion: The short version of the HCA questionnaire demonstrates good validity compared with the longer version. The intake of HCA as assessed with the short questionnaire is comparable to that found in other studies using a short questionnaire.
\end{abstract}

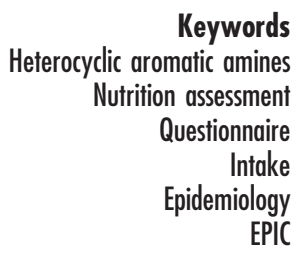

Thirty years ago, mutagenic and carcinogenic compounds were found in fried meat and fish that were called heterocyclic aromatic amines (HCA) because of their chemical structures ${ }^{1}$. HCA are formed from precursors in meat and fish (creatinine, sugar, amino acids) at temperatures exceeding $130^{\circ} \mathrm{C}^{2,3}$. The amount of $\mathrm{HCA}$ production depends mainly on cooking method, temperature, and the meat or fish itself. The highest amounts have been found in foods cooked at high temperatures by methods like barbecuing, grilling and frying ${ }^{2}$. Apart from the meat itself, meat drippings and gravy made from these drippings also contain considerable amounts of $\mathrm{HCA}^{4}$.

Although the carcinogenicity of different HCA has been proved in animal studies ${ }^{5,6}$, conflicting results arose from epidemiological studies. In spite of some case-control studies conducted in Uruguay and the USA showing a possible relationship between the intake of HCA and the risk of breast, colon, lung and gastric cancer ${ }^{7-13}$, other groups were not able to detect any association between HCA intake and cancer risk at different sites ${ }^{14-17}$. Only in some of these studies $8,9,14-16$ was a questionnaire designed to assess the intake of HCA used. In the study of Augustsson et al. ${ }^{16}$, a questionnaire was applied that assessed the degree of browning of foods with the help of photographs, showing the food with different degrees of browning. Voskuil et al. ${ }^{18}$ examined the ability of this HCA questionnaire to assess the intake when the number of items included in the questionnaire was reduced. Their results were encouraging, indicating that the loss of accuracy was small when the number of food items decreased.

It was the purpose of the present study to develop a short questionnaire for the assessment of dietary HCA appropriate for meat and fish consumption habits in Germany. This was done by reducing the list of items of a longer version. In a second step this short questionnaire 
was sent to the participants again and results of both versions were compared.

\section{Methods}

\section{Subjects}

Participants of the European Prospective Investigation into Cancer and Nutrition (EPIC) in Heidelberg were included in this study. EPIC is a multi-centre study focusing on the relation between diet, nutritional and metabolic characteristics, various lifestyle factors and the risk of cancer ${ }^{19}$. In Heidelberg, 25544 persons, aged between 35 and 65 years at the time of recruitment, take part in this study. They were recruited between 1994 and $1998^{20,21}$ and participated in the first follow-up between 1998 and $2000^{22}$. In June 1999, 500 HCA questionnaires were sent to randomly selected participants of EPIC-Heidelberg. Four months later, 385 HCA questionnaires had been returned. After developing the short version of the questionnaire, this was sent again to these 385 participants.

\section{Development of the questionnaire}

The development of the questionnaire was done in two steps. In the first step, the meat and fish items that contributed most to meat and fish intake, as computed from the food-frequency questionnaire (FFQ) used in EPIC-Heidelberg during the recruitment ${ }^{23}$, were chosen for the HCA questionnaire. In addition, food items that were not eaten in high amounts but may contribute much to HCA intake because of high HCA concentration ${ }^{2}$ were included. For each of these food items, the questionnaire addressed different cooking methods usually used in Germany to prepare these foods. For inclusion, the cooking methods had to be relevant for HCA formation. Steaming, boiling, micro-waving and deep-frying do not cause relevant HCA concentrations ${ }^{2,24-26}$, thus only panfrying, broiling and grilling were included in the questionnaire. In total, 26 combinations of food items and cooking methods were considered in the questionnaire (Table 1). The amount of each food item consumed

Table 1 Food items and cooking methods included in the first, longer version of the HCA questionnaire used in a pilot study in EPIC-Heidelberg

\begin{tabular}{ll}
\hline Food item & \multicolumn{1}{c}{ Cooking method } \\
\hline Beef steak, fillet, loin & Pan-fried, broiled, grilled \\
Roast beef, beef roulade, goulash & Pan-fried \\
Pork chop, cutlet, steak, fillet, loin & Pan-fried, broiled, grilled \\
Pork roast, goulash & Pan-fried \\
Bacon, pork belly & Pan-fried, grilled \\
Smoked ham, ribs of pork & Pan-fried, broiled, grilled \\
Meat balls, meat loaf & Pan-fried, broiled \\
Liver loaf ('leberkaese') & Pan-fried, broiled, grilled \\
Bratwurst & Pan-fried, grilled \\
Fried chicken, fried turkey & Pan-fried, broiled, grilled \\
Fish & Pan-fried, broiled, grilled \\
\hline
\end{tabular}

per day was not assessed in the HCA questionnaire, because these data could be obtained from the FFQ applied in the recruitment of EPIC-Heidelberg ${ }^{23,27}$. The amounts of the items consumed were computed by combining consumption frequency and portion size.

Since HCA intake depends on the degree of browning of the foods ${ }^{2}$, participants could indicate which degree of browning they prefer for each of the 11 food items. This was done by means of photographs showing the food items with four different degrees of browning (lightly browned, moderately browned, strongly browned, extremely browned) ${ }^{28}$.

The HCA concentrations in different food items for different cooking methods and degrees of browning were taken from the literature ${ }^{4,29-33}$. By combining information about degree of browning, cooking method and the amount of meat and fish intake, the dietary intake of HCA per day was calculated. HCA intake from gravy was assessed by asking for the use of meat or fish drippings to prepare gravy. When these questions were answered positively, the intake was calculated by combining information on gravy consumption from the FFQ with HCA concentration in gravy of the corresponding meat or fish item and cooking method.

We calculated the HCA intake from this questionnaire for each combination of food item and preparation method, and used these results to develop a short version. A variance-based method, Max_r, was used to reduce the list of food items and preparation methods ${ }^{34,35}$. From a sample of $k$ food items (in this study $k=26$ ), Max_r selects a subset of $L$ foods $(L \ll k)$ that best preserves the between-person variance in nutrient intake. The adequacy of the chosen subset of foods is measured by the Pearson correlation coefficient $r$ between the nutrient intake calculated from the complete list of items and the intake estimated from the subset of items. For selecting the subset of food items, Max_r first chooses the food item that maximises the Pearson correlation coefficient $r$ best; then, excluding this item, the best item from the remaining list is chosen, etc. The results of Max_r were used to reduce the HCA questionnaire. The shorter version thus developed was sent to those participants from whom we had received the first HCA questionnaire.

\section{Statistics}

HCA intake was calculated from the longer and short versions of the HCA questionnaire. The Wilcoxon matched pairs signed rank sum test was used to test for differences in HCA intake calculated from the long and short versions of the questionnaire since data were not normally distributed. Two-tailed $P$-values $<0.05$ were considered to indicate statistical significance. For the total HCA intake as well as for the single HCA, Spearman rank correlation coefficients and their 95\% confidence limits were calculated between both versions of the questionnaire using the SAS procedures PROC FREQ and the options 
Table 2 Ranking of food items and cooking methods according to between-person variance of HCA intake chosen by Max_r $(n=385)$. These food items and cooking methods were used in the short version of the HCA questionnaire

\begin{tabular}{llc}
\hline Number & \multicolumn{1}{c}{ Food item and cooking method } & $\begin{array}{c}\text { Cumulated Pearson } \\
\text { correlation coefficient, } r\end{array}$ \\
\hline 1 & Roast beef, broiled & 0.761 \\
2 & Pork roast, broiled & 0.896 \\
3 & Fried chicken, turkey, grilled & 0.980 \\
4 & Fish, broiled & 0.985 \\
5 & Pork chops, cutlet, fillet, loin, steak, pan-fried & 0.990 \\
6 & Beef steak, fillet, loin, pan-fried & 0.993 \\
7 & Fried chicken, turkey, broiled & 0.995 \\
8 & Fried chicken, turkey, pan-fried & 0.997 \\
9 & Beef steak, fillet, loin, broiled & 0.998 \\
10 & Fish, grilled & 0.999 \\
11 & Pork chops, cutlet, fillet, loin, steak, broiled & 0.999 \\
12 & Ribs of pork, pan-fried & 1.000 \\
\hline
\end{tabular}

MEASURES. This was additionally done for subgroups of the study population regarding sex, age, body mass index (BMI), smoking status, school education and underreporting. BMI was calculated as weight in kilograms divided by height in metres squared and was categorised according to sex and age ${ }^{36}$. Underreporting was defined according to Goldberg et $a l^{37}$ and an energy intake (EI)/basal metabolic rate (BMR) ratio of 0.92 was the cutoff point. Besides Spearman correlation coefficients, the ability of the short version to correctly classify people according to their total HCA intake as well as their intake of the single HCA was measured by cross-classification into quartiles in comparison with the longer version ${ }^{38}$. All statistical analyses were performed using SAS version 6.12 (SAS Institute Inc., Cary, NC, USA).

\section{Results}

The participation rate in this pilot study was $68.8 \%$ (344/500). Among the 344 participants, $45.6 \%$ were men and $54.4 \%$ were women. Their age ranged from 36 to 66 years, with a median of 49 years. All questionnaires had a sufficient quality regarding completeness to be included in the study.

Using the results of the first longer version of the HCA questionnaire of 385 participants, we calculated the contribution to the interpersonal variation of HCA intake of each combination of food item and cooking method. The Pearson correlation coefficient between the HCA intake from broiled roast beef and the total HCA intake calculated from all foods and cooking methods amounted to $r=0.76$ (Table 2). This item explained most of the between-person variance $\left(r^{2}=0.58\right)$. Three combinations of food and cooking method explained more than $90 \%$ of between-person variance in HCA intake. Twelve combinations reached a Pearson correlation coefficient of $r=$ 1.0. These combinations of food items and cooking methods were included in the short questionnaire.

When comparing HCA intake calculated from the long version with the intake calculated from the short version, the results did not differ significantly (Table 3 ). The median intakes of 2-amino-1-methyl-6-phenylimidazo[4,5-b]pyridine (PhIP), 2-amino-3,8-dimethylimidazo[4,5-f]quinoxaline (MeIQx) and 2-amino-3,4,8-trimethylimidazo[4,5- $f$ ]quinoxaline (DiMeIQx) calculated from the short version were

Table 3 5th, 25th, 50th (median), 75th and 95th percentiles, and maximum of HCA intakes (in ng day ${ }^{-1}$ ) calculated from the long and the short versions of the questionnaire $(n=344)$

\begin{tabular}{|c|c|c|c|c|c|c|c|c|}
\hline & \multirow[b]{2}{*}{ Questionnaire } & \multicolumn{5}{|c|}{ Percentile } & \multirow[b]{2}{*}{ Maximum } & \multirow[b]{2}{*}{$P$-value } \\
\hline & & 5 th & 25th & 50th & 75th & 95th & & \\
\hline \multirow[t]{2}{*}{ Total HCA } & Long & 2 & 21 & 89 & 333 & 801 & 10432 & \multirow[t]{2}{*}{0.64} \\
\hline & Short & 2 & 29 & 103 & 303 & 804 & 4194 & \\
\hline \multirow[t]{2}{*}{ PhIP } & Long & 0 & 7 & 53 & 253 & 646 & 9208 & \multirow[t]{2}{*}{0.25} \\
\hline & Short & 0 & 6 & 63 & 225 & 650 & 4060 & \\
\hline \multirow{2}{*}{ MelQx } & Long & 0 & 8 & 25 & 66 & 173 & 1085 & \multirow[t]{2}{*}{0.10} \\
\hline & Short & 1 & 12 & 34 & 118 & 160 & 880 & \\
\hline \multirow[t]{2}{*}{ DiMelQx } & Long & 0 & 0 & 2 & 5 & 25 & 140 & \multirow[t]{2}{*}{0.11} \\
\hline & Short & 0 & 0 & 2 & 4 & 17 & 123 & \\
\hline \multirow[t]{2}{*}{ IQ } & Long & 0 & 0 & 0 & 0 & 3 & 17 & \multirow[t]{2}{*}{0.15} \\
\hline & Short & 0 & 0 & 0 & 1 & 3 & 8 & \\
\hline
\end{tabular}

* Wilcoxon rank sum test.

PhIP-2-amino-1-methyl-6-phenylimidazo[4,5-b]pyridine; MelQx-2-amino-3,8-dimethylimidazo[4,5- $f$ ]quinoxaline; DiMelQx-2-amino-3,4,8-trimethylimidazo $[4,5-f]$ quinoxaline; IQ-2-amino-3-methylimidazo[4,5- $f$ ]quinoline. 
Table 4 Spearman rank correlation coefficients between the HCA intakes computed from the long and short HCA questionnaires and results of quartile cross-classification between HCA intakes calculated from the long and short versions of the questionnaire $(n=344)$

\begin{tabular}{lcccr}
\hline & $\begin{array}{c}\text { Correlation coefficient } \\
\text { (95\% confidence limit) }\end{array}$ & Same quartile & Adjacent quartile & Opposite quartile \\
\cline { 3 - 5 } & $0.51(0.42-0.60)$ & $172(50.0 \%)$ & $113(32.8 \%)$ & $14(4.1 \%)$ \\
Total HCA & $0.46(0.37-0.56)$ & $146(42.4 \%)$ & $140(40.7 \%)$ & $20(5.8 \%)$ \\
PhIP & $0.60(0.52-0.68)$ & $163(47.4 \%)$ & $124(36.0 \%)$ & $5(1.5 \%)$ \\
MelQx & $0.58(0.50-0.66)$ & $169(49.1 \%)$ & $126(36.6 \%)$ & $12(3.5 \%)$ \\
DiMelQx & $0.48(0.38-0.58)$ & NC & NC & NC \\
IQ & &
\end{tabular}

$\mathrm{NC}$ - quartile cross-classification was not calculated for IQ.

63, 34 and 2 ng day $^{-1}$, respectively. The median intake of total HCA amounted to $102 \mathrm{ng} \mathrm{day}^{-1}$ as assessed with the short version, with a range from 0 to $4194 \mathrm{ng} \mathrm{day}^{-1}$. The range in the longer version was larger, but in both versions the 95th percentile of total HCA intake was about 800 ng day $^{-1}$. PhIP contributed most to the total HCA intake, followed by MeIQx.

Spearman rank correlation coefficient between the long and the short versions was 0.5 for total HCA, 0.46 for PhIP, 0.58 for DiMeIQx and 0.6 for MeIQx. All correlations were significant at $P<0.001$ (Table 4). Fig. 1 shows the relationship between the total HCA intakes calculated from the long and short versions of the questionnaire in detail. When looking at the correlation coefficients of subgroups, some (although not statistically significant) differences could be observed (Table 5). The correlation coefficient was higher for men than for women and an increasing correlation was observed for an increasing number of years of school education. Among persons with different BMI, no tendency could be observed. In quartile cross-classification between the long and the short versions of the questionnaire, 42.4\% (PhIP) and 50.0\% (total HCA) were assigned to the same quartile of intake (Table 4). Between 32.8\% and 40.7\% were classified into

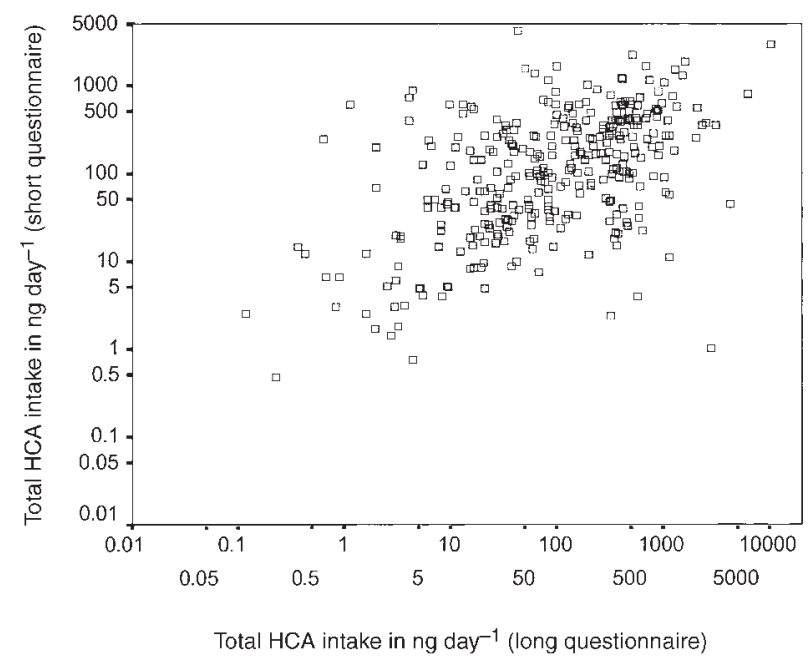

Fig. 1 Scatter plot of total HCA intakes calculated from the long and short versions of the HCA questionnaire (logarithmic scale) an adjacent quartile and gross misclassification amounted to $5.8 \%$ of the participants.

\section{Discussion}

The aim of this pilot study was the development of a short questionnaire for the assessment of HCA intake. Instruments for the assessment of these substances are needed since several studies that investigated the association between HCA intake and the cancer risk of different sites gave conflicting results. A recently published US study indicates a higher risk of breast cancer with an increasing intake of PhIP', while an older study was not able to demonstrate any association ${ }^{15}$. Furthermore, two studies point at a higher risk of lung cancer and colorectal adenomas, respectively, and the intake of MeIQx ${ }^{7,8}$. Unlike several older studies ${ }^{10-13}$, these studies used a specially designed questionnaire ${ }^{39}$. In these older studies instead, assumptions about the frequency of cooking

Table 5 Spearman rank correlation coefficients between total HCA intakes computed from the long and short HCA questionnaires for different subgroups of the study population $(n=344)$

\begin{tabular}{|c|c|c|c|}
\hline Characteristic & Subgroup & $n$ & $\begin{array}{c}\text { Correlation } \\
\text { coefficient } \\
\text { (95\% confidence } \\
\text { limits) }\end{array}$ \\
\hline \multirow[t]{2}{*}{ Sex } & Female & 186 & $0.49(0.36-0.62)$ \\
\hline & Male & 158 & $0.55(0.42-0.68)$ \\
\hline \multirow[t]{3}{*}{ Age group (years) } & $35-44$ & 96 & $0.46(0.27-0.64)$ \\
\hline & $45-54$ & 139 & $0.56(0.42-0.69)$ \\
\hline & $55+$ & 109 & $0.49(0.31-0.66)$ \\
\hline \multirow[t]{3}{*}{ School education } & $\leq 8$ years & 120 & $0.39(0.21-0.57)$ \\
\hline & 10 years & 70 & $0.49(0.29-0.69)$ \\
\hline & High school degree & 154 & $0.56(0.43-0.68)$ \\
\hline \multirow[t]{2}{*}{ Underreporting* } & Underreporter & 41 & $0.60(0.37-0.83)$ \\
\hline & Non-underreporter & 303 & $0.49(0.39-0.59)$ \\
\hline \multirow[t]{4}{*}{$\mathrm{BMI}$} & Underweight & 60 & $0.67(0.48-0.85)$ \\
\hline & Normal & 175 & $0.43(0.29-0.57)$ \\
\hline & Overweight & 60 & $0.56(0.35-0.76)$ \\
\hline & Obese & 49 & $0.48(0.26-0.70)$ \\
\hline \multirow[t]{3}{*}{ Smoking status } & Non-smoker & 146 & $0.53(0.39-0.66)$ \\
\hline & Ex-smoker & 112 & $0.53(0.37-0.69)$ \\
\hline & Smoker & 86 & $0.45(0.25-0.65)$ \\
\hline
\end{tabular}

* Underreporting defined as El/BMR $<0.92$ (in accordance with Goldberg et al. ${ }^{37}$ ). 
methods used in the study population and the preferred degree of browning were made. This probably introduces a bias in the estimation of HCA intake. Since a questionnaire used for nutrient assessment should be as short, but also as accurate as possible, Voskuil and coworkers ${ }^{18}$ evaluated the possibility to reduce the number of food items used in questionnaires for HCA assessment. They were able to demonstrate that the resulting loss of accuracy was negligible when the number of dishes was reduced from 39 to 15 .

Our study was conducted in a similar way. In the first step of the development of the questionnaire, a longer version including the most important meat and fish items was developed. This version was reduced in a second step and, in addition to what Voskuil et al. ${ }^{18}$ did in their study, validated in a field study.

For calculating HCA intakes no analyses of meat and fish samples prepared by different cooking methods were performed. Instead, data from the literature were used $^{29-33}$. We selected those data that were analysed in a standardised way and were most likely to be appropriate for the situation in Germany. Nevertheless, this approach may be a source of bias. Firstly, the HCA concentration in the foods included in the questionnaire in Heidelberg might be higher or lower than the concentration in the foods used in the literature, although the foods look similar in the pictures used to indicate the degree of browning. Secondly, HCA concentration in typical German foods, e.g. liver loaf ('leberkaese'), had to be assumed because no corresponding data were available. In these cases, data for foods that were similar regarding fat, water and protein contents were chosen, but this does not automatically imply that HCA content is approximately the same. In conclusion, using literature data on HCA concentration in meat and fish to estimate the intake may introduce an over- or underestimation. This is important when the aim of a questionnaire is to assess the intake of a nutrient quantitatively. If the purpose of a questionnaire, especially of a short questionnaire, is to assess the variance of the intake and to categorise individuals according to their intake, an over- or underestimated intake is negligible if the bias is nondifferential. Non-differential misclassification is often a problem inherent in data collection methods ${ }^{40}$.

Included in the longer version of the questionnaire were those food items that are eaten in high amounts according to the results of the recruitment FFQ. Additionally, food items that were eaten less frequently in this cohort, e.g. bacon, were included because they can contribute much to HCA intake due to the high HCA concentration in the prepared food ${ }^{2}$. Using the variance-based method Max_r ${ }^{34,35}$, the list of food items and cooking methods was reduced. The shorter version of the HCA questionnaire included those food items that contributed most to between-person variance of total HCA intake. In our study, one cooked food item (broiled roast beef) explained $58 \%$ of between-person variance of total HCA intake, which is comparable to the results of Voskuil et al. $^{18}$. In their analyses, fried pork chops explained $48 \%$ of between-person variance. Willett ${ }^{41}$ suggested that a short questionnaire should include at least as many food items as are used to explain $80 \%$ of the variation of nutrient intake. We included all items that explained 99\% of the variance of HCA intake. Since two items explain more than $80 \%$ of the variance, this will not give a detailed picture of important meat items and cooking methods used in Germany. For that reason 12 combinations of food items and cooking methods were included in the questionnaire.

The calculated intake of $103 \mathrm{ng}$ HCA per day is comparable to the intake computed in Swedish studies $^{16,28}$. They calculated for different groups of participants a median intake varying from 77 to 160 ng day $^{-1}$. PhIP contributed a higher proportion to HCA intake in the Heidelberg study than in the Swedish one $^{28}$. The results from Heidelberg concerning PhIP intake are more comparable with results from US studies ${ }^{39}$. US data $^{31}$ were used to calculate HCA intake from grilled chicken in our pilot study because no data from Sweden were available ${ }^{28}$. Therefore, grilled chicken contributes much to PhIP intake in the present study. In the Swedish study, HCA concentration of pan-fried chicken was used for grilled chicken, which might underestimate HCA intake. However, using US data for grilled chicken in a German study possibly overestimates HCA concentration, since Americans may prefer eating meat darker than Germans.

In the study conducted in Heidelberg, correlation coefficients between the long and short versions of the HCA questionnaire are 0.46 or higher, which is an acceptable result ${ }^{42}$. Although correlation coefficients in the subgroups do not differ significantly (overlapping confidence limits), there are some interesting differences. Increasing correlation coefficients with increasing educational level might a hint at more accurate filling in of questionnaires by better-educated subjects. The differing correlation coefficient between underreporters and nonunderreporters can be due to chance alone, because of the strata size. However, underreporters might be more precise in reporting the way in which they prepare their meals. The results of the quartile cross-classification of the Heidelberg pilot study are in the range of other studies comparing a short questionnaire with a longer ver$\operatorname{sion}^{43,44}$. However, one must keep in mind that the longer HCA questionnaire used as a reference instrument cannot be considered as a gold standard. We do not believe that the long questionnaire reflects the true HCA intake in the study population, but our assumption behind this kind of questionnaire development and validation is that the long version of the questionnaire assesses the HCA intake more accurately than the short version because it includes more food items and cooking methods. Therefore, we would like to know and test whether a version with a reduced 
number of items assesses the intake as well as the long version without losing too much accuracy. The similarities in design of the longer and the short versions of the questionnaire may cause an overestimated validity of the short version $^{41}$. No gold standard exists to validate an HCA questionnaire, since measurements of DNA-adducts ${ }^{45,46}$ or excreted HCA metabolites in urine ${ }^{47,48}$ are either not sensitive enough or have other limitations. An appropriate way could be the double portion technique, where a part of the food consumed is analysed for its HCA content, but this method is expensive and only useful in small groups of individuals.

In conclusion, the developed short version of an HCA questionnaire demonstrates good validity in comparison with the longer, more extensive version. The total intake of HCA as assessed with the short questionnaire is consistent with the exposure in other studies using a short questionnaire. According to these results, the short questionnaire can be used to further examine whether HCA intake is related to cancer risk. Since HCA exposure varies depending on cooking methods and the level of cooked meat consumption, EPIC offers the opportunity to obtain information on cooking of meat and fish in different regions of Europe with a variety of consumption habits. Using the questionnaire at least in some of these countries and regions provides a good chance to proceed in our knowledge on the association between HCA intake, cooking methods and cancer risk.

\section{Acknowledgements}

We would like to thank all participants of EPICHeidelberg, as well as Dr Gunnar Steineck and Dr Katarina Augustsson for allowing us to use their photos for assessment of the degree of browning of different foods. EPIC-Heidelberg is supported by Deutsche Krebshilfe, Deutsches Krebsforschungszentrum and the programme 'Europe against Cancer' of the European Commission.

\section{References}

1 Sugimura T. Overview of carcinogenic heterocyclic amines. Mut. Res. 1997; 376: 211-9.

2 Skog K, Johansson MAE, Jägerstad MJ. Carcinogenic heterocyclic amines in model systems and cooked foods: a review on formation, occurrence and intake. Food Chem. Toxicol. 1998; 36: 879-96.

3 Layton DW, Bogen KT, Knize MG, et al. Cancer risk of heterocyclic amines in cooked foods: an analysis and implications for research. Carcinogenesis 1995; 16: 39-52.

4 Gross GA, Grüter A. Quantification of mutagenic/carcinogenic heterocyclic aromatic amines in food products. J. Chromatogr. 1992; 592: 271-8.

5 Adamson RH, Thorgeirsson UP, Snyderwine EG, et al. Carcinogenicity of 2-amino-3-methylimidazo[4,5- $f$ ]quinoline in nonhuman primates: induction of tumors in three macaques. Jpn. J. Cancer Res. 1990; 81: 10-4.

6 Shirai $\mathrm{T}$, Tamano S, Sano $\mathrm{M}$, et al. Carcinogenicity of 2-amino-1-methyl-6-phenylimidazo[4,5-b]pyridine (PhIP) in rats: dose-respose studies. Princess Takamatsu Symposia 1995; 23: 232-9.

7 Sinha R, Kulldorff M, Chow W-H, et al. Dietary intake of heterocyclic amines, meat-derived mutagenic activity, and risk of colorectal adenomas. Cancer Epidemiol. Biomark. Prev. 2001; 10: 559-62.

8 Sinha R, Kulldorff M, Swanson CA, et al. Dietary heterocyclic amines and the risk of lung cancer among Missouri women. Cancer Res. 2000; 60: 3753-7.

9 Sinha R, Gustafson DR, Kulldorf M, et al. 2-Amino-1-methyl6-phenylimidazo[4,5-b]pyridine, a carcinogen in hightemperature-cooked meat, and breast cancer risk. J. Natl. Cancer Inst. 2000; 92: 1352-4.

10 De Stefani E, Boffetta P, Mendilaharsu M, et al. Dietary nitrosamines, heterocyclic amines, and risk of gastric cancer: a case-control study in Uruguay. Nutr. Cancer 1998; 30: 158-62.

11 De Stefani E, Ronco A, Mendilaharsu M, et al. Case-control study on the role of heterocyclic amines in the atiology of upper aerodigestive cancers in Uruguay. Nutr. Cancer 1998; 32: $43-8$.

12 De Stefani E, Ronco A, Mendilaharsu M, et al. Meat intake, heterocyclic amines, and risk of breast cancer: a casecontrol study in Uruguay. Cancer Epidemiol. Biomark. Prev. 1997; 6: 573-81.

13 De Stefani E, Doneo-Pellegrini H, Mendilaharsu M, et al. Meat intake, heterocyclic amines and risk of colorectal cancer: a case-control study in Uruguay. Int. J. Oncol. 1997; 10: $573-80$.

14 Norrish AE, Ferguson LR, Knize MG, et al. Heterocyclic amine content of cooked meat and risk of prostate cancer. J. Natl. Cancer Inst. 1999; 91: 2038-44.

15 Delfino RJ, Sinha R, Smith C, et al. Breast cancer, heterocyclic amines from meat and $N$-acetyltransferase 2 genotype. Carcinogenesis 2000; 21: 607-15.

16 Augustsson K, Skog K, Jägerstad $\mathrm{M}$, et al. Dietary heterocyclic amines and cancer of the colon, rectum, bladder, and kidney: a population-based study. Lancet 1999; 353: 703-7.

17 Lyon JL, Mahoney AW. Fried foods and the risk of colon cancer. Am.J. Epidemiol. 1988; 128: 1000-6.

18 Voskuil DW, Augustsson K, Dickman PW, et al. Assessing the human intake of heterocyclic aromatic amines: limited loss of information using reduced sets of questions. Cancer Epidemiol. Biomark. Prev. 1999; 8: 809-14.

19 Riboli E, Kaaks R. The EPIC project: rationale and study design. Int. J. Epidemiol. 1997; 26(1): S6-14.

20 Boeing $\mathrm{H}$, Wahrendorf J, Becker N. EPIC-Germany - a source for studies into diet and risk of chronic diseases. Ann. Nutr. Metab. 1999; 43: 195-204.

21 Boeing H, Korfmann A, Bergmann MM. Recruitment procedures of EPIC-Germany. Ann. Nutr. Metab. 1999; 43: 205-15.

22 Bergmann M, Bussas U, Boeing H. Follow-up procedures in EPIC-Germany - data quality aspects. Ann. Nutr. Metab. 1999; 43: 225-34

23 Brandstetter BR, Korfmann A, Bergmann MM. Dietary habits in the German EPIC cohorts: food group intake estimated with food frequency questionnaire. Ann. Nutr. Metab. 1999; 43: $246-57$.

24 Zhang X-M, Wakabayashi K, Liu Z-C, et al. Mutagenic and carcinogenic heterocyclic amines in Chinese cooked foods. Mut. Res. 1988; 201: 181-8.

25 Taylor SL, Berg CM, Shoptaugh NH, et al. Lack of mutagens in deep-fat fried foods obtained at the retail level. Food Chem. Toxicol. 1982; 20: 209-12.

26 Nilsson L, Övervik E, Fredholm L, et al. Influence of frying fat on mutagenic activity in lean pork meat. Mut. Res. 1986; 171: $115-21$.

27 Bohlscheid-Thomas S, Hoting I, Boeing $\mathrm{H}$, et al. 
Reproducibility and relative validity of energy and macronutrient intake of a food frequency questionnaire developed for the German part of the EPIC project. Int. J. Epidemiol. 1997; 26(Suppl 1): S71-81.

28 Augustsson K, Skog K, Jägerstad M, et al. Assessment of the human exposure of heterocyclic amines. Carcinogenesis 1997; 18: 1931-5.

29 Abdulkarim BG, Smith JS. Heterocyclic amines in fresh and processed meat products. J. Agric. Food Chem. 1998; 46: 4680-7.

30 Sinha R, Knize MG, Salmon CP, et al. Heterocyclic amine content of pork products cooked by different methods and by varying degrees of doneness. Food Chem. Toxicol. 1998; 36: 289-97.

31 Sinha R, Rothman N, Brown ED, et al. High concentrations of the carcinogen 2-amino-1-methyl-6-phenylimidazo[4,5$b$ ]pyridine [PhIP] occur in chicken but depending on the cooking temperature. Cancer Res. 1995; 55: 4516-9.

32 Skog K, Augustsson K, Steineck G, et al. Polar and non-polar heterocyclic amines in cooked fish and meat products and their corresponding pan residues. Food Chem. Toxicol. 1997; 35: $555-65$.

33 Skog K, Steineck G, Augustsson K, et al. Effect of cooking temperature on the formation of heterocyclic amines in fried meat products and pan residues. Carcinogenesis 1995; 16: $861-7$.

34 Mark SD, Thomas DG, Decarli A. Measurement of the exposure to nutrients: an approach to the selection of informative foods. Am. J. Epidemiol. 1996; 143: 514-21.

35 Thomas DG, Mark SD. Max_r: an optimal method for the selection of subsets of foods for the measurement of specific nutrient exposures. Comp. Meth. Prog. Biomed. 1997; 54: $151-6$.

36 National Research Council. Diet and Health: Implications for Reducing Chronic Disease Risk. Washington, DC: National Academy Press, 1989.

37 Goldberg GR, Black AE, Jebb SA, et al. Critical evaluation of energy intake data using fundamental principles of energy physiology: 1 . Derivation of cut-off limits to identify underrecording. Eur. J. Clin. Nutr. 1991; 45: 569-81.

38 Garrow JS. Validation of methods for estimating habitual diets: proposed guidelines. Eur. J. Clin. Nutr. 1995; 49: 231-2.

39 Byrne C, Sinha R, Platz EA, et al. Predictors of dietary heterocyclic amines in three prospective cohorts. Cancer Epidemiol. Biomark. Prev. 1998; 7: 523-9.

40 Gordis L. Epidemiology. Philadelphia, PA: WB Saunders Company, 1996.

41 Willett WC. Nutritional Epidemiology, 2nd ed. New York: Oxford University Press, 1998.

42 Block G, Hartman AM. Issues in reproducibility and validity of dietary studies. Am. J. Clin. Nutr. 1989; 50: 1133-8.

43 Dobson AJ, Blijlevens R, Alexander HM, et al. Short fat questionnaire: a self-administered measure of fat-intake behaviour. Aust. J. Public Health 1993; 17: 144-9.

44 Bingham SA, Gill C, Welch A, et al. Comparison of dietary assessment methods in nutritional epidemiology: weighed records v. $24 \mathrm{~h}$ recalls, food-frequency questionnaires and estimated-diet records. Br.J. Nutr. 1994; 72: 619-43.

$45 \mathrm{Ji} \mathrm{H}$, Yu MC, Stillwell WG, et al. Urinary excretion of 2-amino-3,8-dimethylimidazo[4,5- $f$ ]quinoxaline in white, black, and Asian men in Los Angeles County. Cancer Epidemiol. Biomark. Prev. 1994; 3: 407-11.

46 Kidd LCR, Stillwell WG, Yu MC, et al. Urinary excretion of 2-amino-1-methyl-6-phenylimidazo[4,5- $b$ ]pyridine (PhIP) in white, African-American, and Asian-American men in Los Angeles County. Cancer Epidemiol. Biomark. Prev. 1999; 8: 439-45.

47 Mauthe RJ, Dingley KH, Leveson SH, et al. Comparison of DNA-adduct and tissue-available dose levels of MeIQx in human and rodent colon following administration of a very low dose. Int. J. Cancer 1999; 80: 539-45.

48 Wakabayashi K, Totsuka Y, Fukotome K, et al. Human exposure to mutagenic/carcinogenic heterocyclic amines and co-mutagenic $\beta$-carbolines. Mut. Res. 1997; 376: 253-9. 